asked to complete questionnaires recording their IBS symptom severity (IBS SSS), quality of life, non-colonic symptoms, anxiety and depression before and after treatment. In addition, they completed questionnaires detailing their perception of HT, other people's perception of HT and their expectations about the efficacy of HT. Their perceptions about the hypnotherapeutic process were assessed both quantitatively and also qualitatively using patient descriptions. Furthermore, the analysis compared the characteristics of responders and nonresponders.

Results 39 out of 50 patients $(78 \%, \mathrm{p}<0.001)$ responded to treatment (50 point or more reduction in IBS SSS), which is exactly consistent with our previously published data. Pain scores, non-colonic symptoms, quality of life, anxiety and depression also significantly improved after HT (all p<0.001). When asked how patients felt before treatment, $52 \%$ of responses portrayed hypnotherapy negatively compared to 3\% after treatment. The relatives and doctors of patients were generally supportive of HT although one cognitive behavioural therapist advised against it. In responders, 19 patients $(48.7 \%)$ expected hypnotherapy to be effective prior to starting it, whereas in non-responders, 7 (64\%) expected treatment to be successful. Interestingly, 9 of 11 non-responders (82\%) considered treatment worthwhile despite no significant effect on their symptoms. This may be because 46 patients $(92 \%$ of all patients) had found HT helped them with other issues, such as dealing with stressful situations or poor sleep.

Conclusion Although initially being perceived negatively, hypnotherapy significantly improved symptoms and resulted in other benefits not related to the gastrointestinal system. Interestingly, those with greater expectation about treatment did not seem to do quite so well, suggesting that high expectations are not necessary for a good outcome.

\section{PWE-129 GENDER DIFFERENCES IN PATIENTS WITH CHRONIC CONSTIPATION IN A TERTIARY REFERRAL CLINIC}

${ }^{1}$ Christopher Emmett*, 'Linda Tinkler, ${ }^{2}$ Usman Khan, ${ }^{1}$ Yan Yiannakou. ${ }^{1}$ County Durham And Darlington NHS Foundation Trust, UK; ${ }^{2}$ Wrexham Maelor Hospital, Wrexham, UK

\subsection{6/gutjnl-2018-BSGAbstracts.423}

Introduction Chronic constipation (CC) is a very common symptom, and is more commonly seen in women. This difference is much more pronounced in secondary care. It is possible that the excess prevalence to secondary care is due to greater severity or to differences in healthcare seeking. This study aims to evaluate the differences in the characteristics of constipation seen in male and female patients attending a tertiary referral clinic.

Methods The study was designed as a prospective cohort study running at a single tertiary referral clinic. Data were collected prospectively from eligible patients following informed consent using a standardised proforma, and entered into a study database. Patients were categorised according to the Rome III criteria as having Functional Constipation (FC) or Irritable Bowel Syndrome- Constipation type (IBS-C), and were asked to complete PAC-SYM and PAC-QOL questionnaires

Results Data were collected from 827 patients between January 2007 and October 2014; of these, 766 patients were included. Of these, $103(13 \%)$ were male. The mean age at presentation was 42 years; for males this was 49.6 years, compared to 41.1 years for females; a mean difference of 8.4 years $(p<0.0001)$. Women were significantly younger at onset of symptoms, with a mean age of 23 years at onset of symptoms compared to 34 years for men, a mean difference of 11 years $(p<0.0001)$. There was no significant difference in mean duration of symptoms at presentation between the sexes. Women reported significantly higher PAC-SYM and PAC-QOL scores than men. A ratio of QOL:SYM was used as a marker for coping strategies. Women had a higher mean ratio (1.12 vs 0.39) however this was non-statistically significant $(\mathrm{p} \geq 0.239)$

Conclusions This study reports a similar male:female ratio to other studies, with far more women than men. It also suggests that women experience symptom onset at a younger age, and that they are also younger when they present. Female patients report significantly more severe symptoms and significantly worse quality of life, with significantly higher mean PAC-SYM and PAC-QOL scores. Although QOL:SYM ratio was higher in women, suggesting greater impact of milder symptoms on QOL, this was not significant. The reasons for these differences are not clear, and further work is needed in this area

\section{PWE-130 SIBO CONFERS HEIGHTENED SYMPTOMS BURDEN IN IBS, IN THE ABSENCE OF CHANGES IN GASTROINTESTINAL TRANSIT}

${ }^{1}$ Adam Farmer*, ${ }^{2}$ Anthony Hobson. ' University Hospitals Of North Midlands, Stafford, UK; ${ }^{2}$ Functional Gut Clinic, London, UK

10.1136/gutjnl-2018-BSGAbstracts.424

Introduction Although controversial, small intestinal bacterial overgrowth (SIBO) has been associated with irritable bowel syndrome (IBS) (1). However, little is known regarding the effect of concomitant SIBO in patients with IBS in terms of symptom burden, quality of life or its effect on gastrointestinal (GI) motility. We aimed to compare the effect of SIBO on symptom burden, quality of life and segmental/panenteric motility in IBS.

Methods 27 patients with Rome III defined IBS-mixed bowel habit (IBS-M) (3 male, mean age 36.5 years, range 18-65) underwent a wireless motility capsule (WMC) using a standardised protocol. The WMC concurrently measures $\mathrm{pH}$, pressure and temperature as it traverses the GI tract. Segmental transit was derived from measures around known anatomical landmarks as identified by compartmental $\mathrm{pH}$ changes. Ileal and colonic motility measures are presented as area under the curve (AUC) derived from contraction amplitude and frequency. Validated questionnaires assessing GI (verbal descriptor anchored visual analogue scale (VDVAS) assessing sensory intensity (VDVAS-I) and unpleasantness (VDVAS-U)), somatic symptoms (Personal Health Questionnaire (PHQ) and quality of life (EQ-5 D) were administered. A standardised lactulose hydrogen breath test was subsequently performed and interpreted according to recently published guidelines (2).

Results 14/27 patients (51.8\%) were positive for SIBO based on breath testing. Changes in GI motility between SIBO positive and negative patients are shown in Table 1 . Patients with concomitant SIBO had higher VDVAS-I and VDVAS-U (147 \pm 21 vs. $127 \pm 20, \quad \mathrm{p} \geq 0.048$ and $135 \pm 9.2$ vs. $109 \pm 6.2$, $\mathrm{p} \geq 0.02)$ and somatic symptoms $(9.8 \pm 3.2$ vs. $7.3 \pm 2.3$, $\mathrm{p} \geq 0.03$ ). SIBO positive patients had reduced quality of life in comparison to those without (43.2 \pm 16 vs. $60 \pm 15, \mathrm{p} \geq 0.008)$. 